\title{
Rarely seen radial nerve total injury with humerus supracondylar type IIIA open fracture in a child: Our five-year follow-up results
}

\author{
Duran Topak (D), Ökkeş Bilal(i), Fatih Doğar (D), Mustafa Abdullah Özdemir (D) \\ Department of Orthopedics and Traumatology, Kahramanmaraş Sütçü Imam University Faculty of Medicine, Kahramanmaraş, Turkey
}

Supracondylar humerus fractures are one of the most frequent fractures in children, accounting for 3 to $18 \%$ of all pediatric fractures and more than $60 \%$ of pediatric fractures of the elbow. ${ }^{[1]}$

Some serious complications can accompany supracondylar humerus fractures. Early complications are neurological and vascular injuries, compartment syndrome, and muscle damage. ${ }^{[2,3]}$ Incidences as high as $20 \%$, and 2 to $3 \%$ have been reported for traumatic and iatrogenic nerve injuries, respectively, associated with supracondylar humerus fractures. About 4.1\% of extension-type supracondylar humerus fractures have traumatic radial nerve lesions. ${ }^{[4]}$

Herein, we present a rarely seen eight-year-old patient diagnosed with a Gustilo-Anderson type IIIA open, Gartland type IV supracondylar left humerus

Received: November 01, 2021

Accepted: December 19, 2021

Published online: December 22, 2021

Correspondence: Duran Topak, MD. Kahramanmaraş

Sütçü İmam Üniversitesi Tıp Fakültesi, 46040 Onikişubat,

Kahramanmaraş, Türkiye.

E-mail: drdtopak@gmail.com

Doi: 10.52312/jdrscr.2022.17

Citation: Topak D, Bilal Ö, Doğar F, Özdemir MA. Rarely seen radial nerve total injury with humerus supracondylar type IIIA open fracture in a child: Our five-year follow-up results. Jt Dis Relat Surg Case Rep 2022;1(1):39-42.

C2022 All right reserved by the Turkish Joint Diseases Foundation

This is an open access article under the terms of the Creative Commons Attribution-NonCommercial License, which permits use, distribution and reproduction in any medium, provided the original work is properly cited and is not used for commercial purposes (http://creativecommons.org/licenses/by-nc/4.0/).

https://www.casereportsjointdrs.org

\section{ABSTRACT}

Supracondylar humerus fractures, typically caused by falling with an arm outstretched and landing on an open palm, are among the most common childhood injuries. We present a rarely seen eight-year-old patient diagnosed with a Gustilo-Anderson type IIIA open, Gartland type IV left supracondylar humerus fracture with radial nerve total injury in contact with the external environment where the injury nerve endings were clean. The open humerus supracondylar type IIIA fracture was treated by open reduction and fixation with performed using Kirschner wires and the radial nerve was repaired using microsurgical techniques. In conclusion, excellent functional and cosmetic outcomes can be achieved clinically by surgical debridement, irrigation with saline solutions, and appropriate antibiotherapy as prophylaxis for an unstretched primary repair of the radial nerve in an open supracondylar humerus fracture that exposed the radial nerve.

Keywords: Humerus supracondylar fracture, microsurgery open fracture, radial nerve injury.

fracture with radial nerve total injury in contact with the external environment, where the injury nerve endings were clean and a five-year follow-up of the radial nerve after microsurgical repair.

\section{CASE REPORT}

An eight-year-old boy who fell while cycling was applied to our emergency service at 07:45 P.M. with an open left elbow fracture and was operated at 11:00 P.M. A physical examination revealed an approximately $8-\mathrm{cm}$ transverse subcutaneous skin and muscle incision starting from the anterolateral region of the humeral distal to the posteromedial region. Manual palpation of the distal pulse of the patient and distal capillary circulation revealed normal blood flow. The left humeral distal bone was detached from 

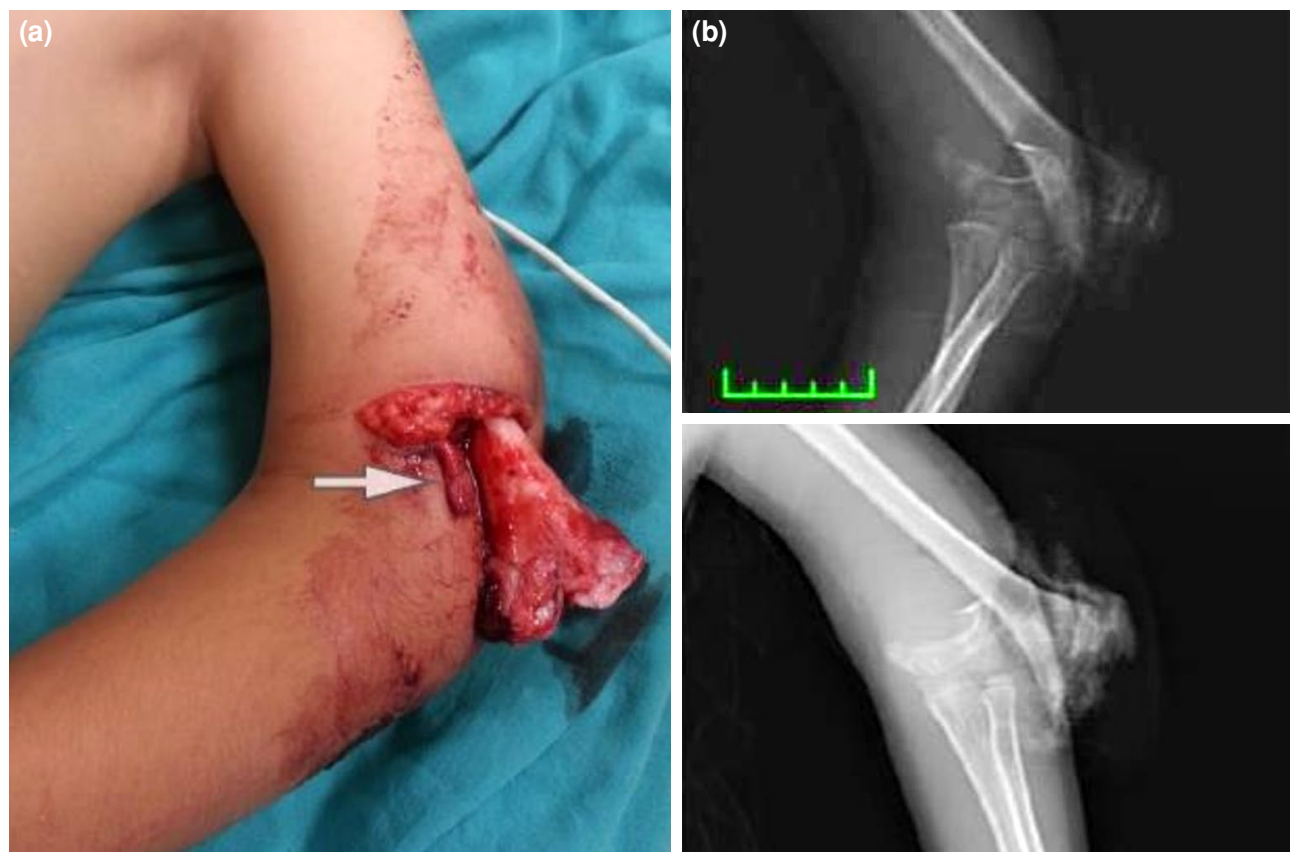

Figure 1. (a) Supracondylar humerus fracture classified as type IIIA according to the GustiloAnderson criteria. The radial nerve is visible and marked with a white arrow. (b) Preoperative X-ray of our patient classified as a Gartland criteria type IV and Gustilo-Anderson criteria type IIIA.

the periosteum and the radial nerve was totally cut. The left humeral distal region and the radial nerve were in contact with the external environment (Figure 1a). Radiographs were taken of the patient's elbow, and the patient was evaluated as having a Gustilo Anderson type IIIA and modified Gartland type IV left supracondylar humerus fracture, and the direction of the distal part was posteromedial (Figure 1b).

With a body weight of $25 \mathrm{~kg}$ and height of $126 \mathrm{~cm}$, the patient was administered cefazolin sodium at
25 to $50 / \mathrm{kg}$ dose, divided into three equal doses, combined with gentamycin sulfate at a 3 to $6 \mathrm{mg} / \mathrm{kg}$ dose, once a day during the day before and after surgery. ${ }^{[5]}$ A neurovascular examination revealed a radial nerve deficiency. After primary wound care and evaluation, the patient was taken immediately to the operating room. During the debridement and exploration of the wound site, the radial nerve was confirmed as injury totally and cleanly.

After irrigation with isotonic saline and debridement, the fractured humerus was reduced
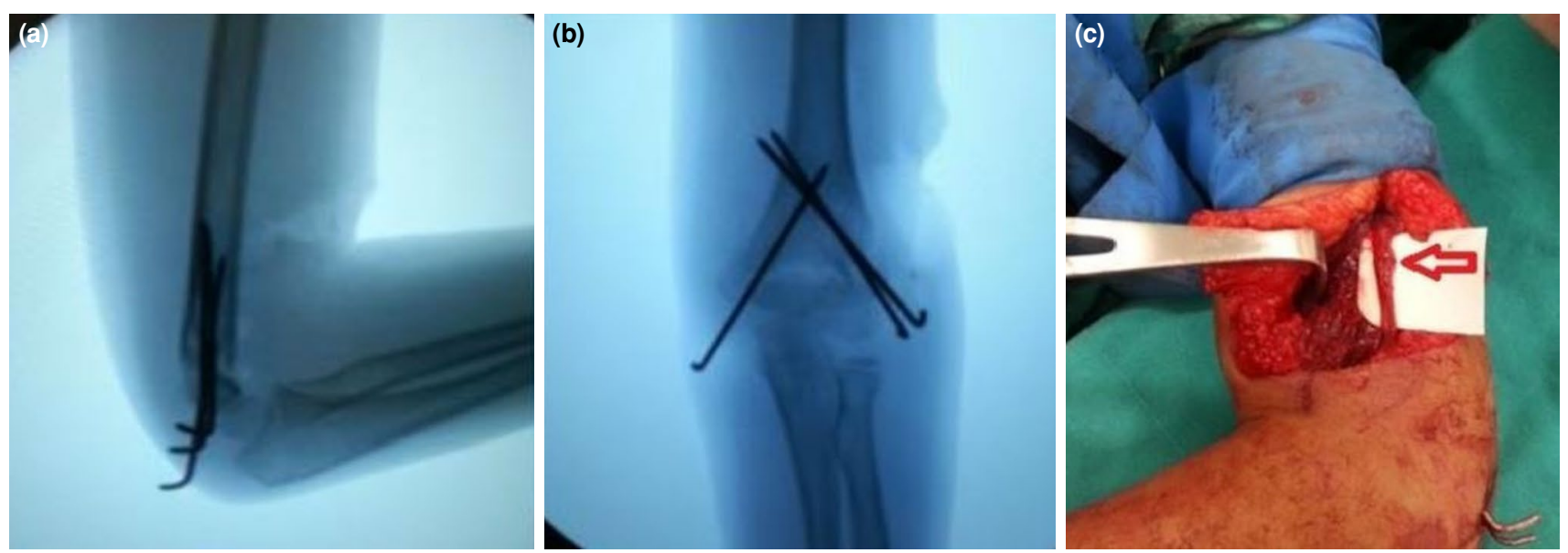

Figure 2. (a, b) X-ray of a supracondylar humerus fracture fixated with two K-wires placed laterally and one K-wire placed medially. (c) Intraoperative view of the primary repair of the radial nerve. 

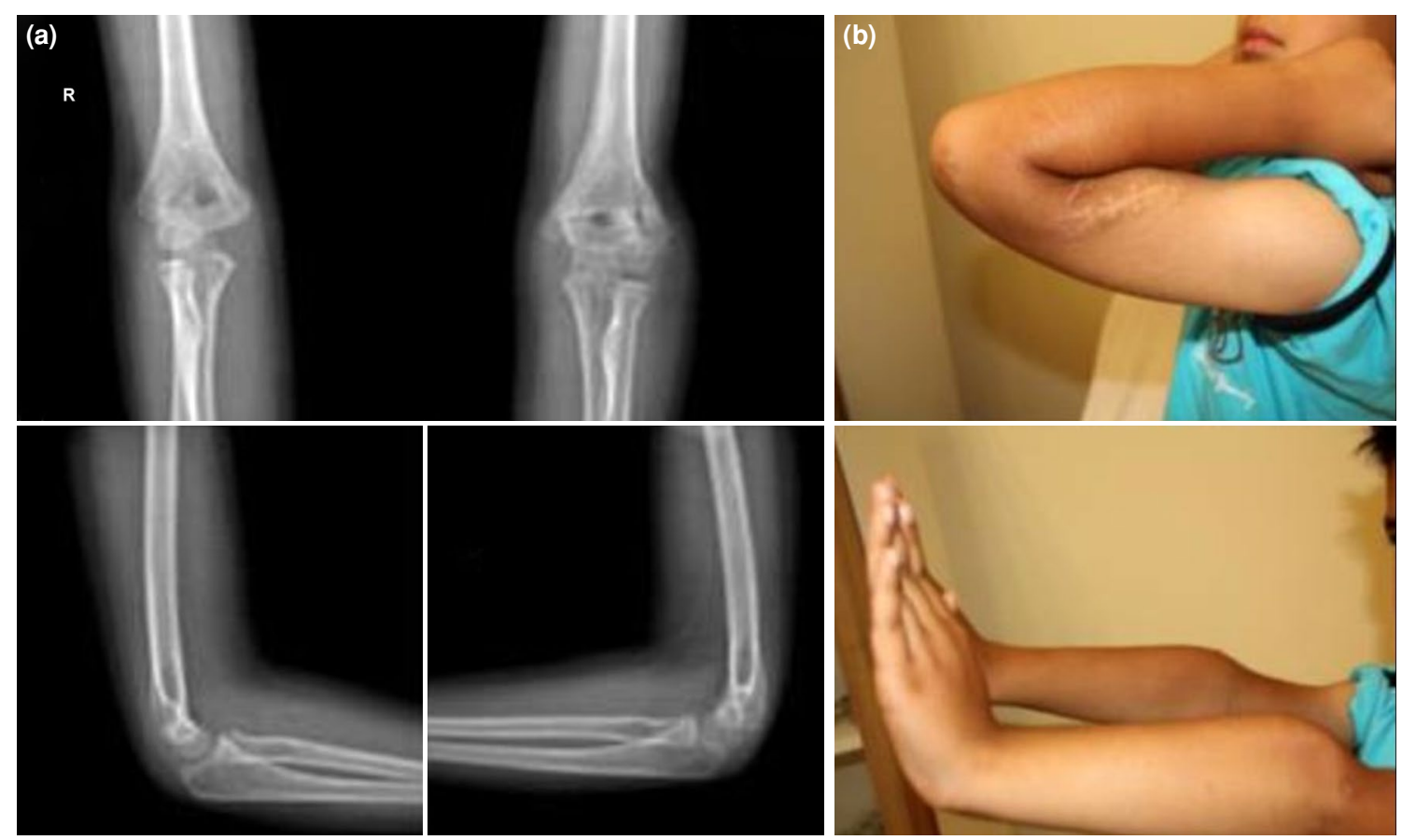

Figure 3. (a) Anteroposterior and lateral X-ray images of left and right elbow at one-year follow-up. (b) Full flexion and extension of elbow of our patient at five-year postoperative follow-up.

openly. Fixation was made with one medial and two lateral crossed Kirschner-wires (K-wires) under a C-armed fluoroscope (Figure 2a).

When the injury radial nerve endings were brought to the tip for primary repair, they appeared to be unstretched. The radial nerve was repaired under microsurgical conditions with 9.0 and 10.0 monofilament suture materials (Figure 2b). After three days of observation and antibiotic administration, the patient was discharged. After three to four weeks of follow-up, the long arm plaster and K-wires were removed and the patient started to exercise the arm. After one year, radiographs displayed complete fracture healing (Figure 3a). At the end of the fifth year of follow-up, the patient's physical examination showed full elbow active range of motion on flexion and extension and full range of motion of the wrist and finger extension, with complete muscle power $(5 / 5)$ for the injured left upper extremity muscles, sensory examination and humerus length was normal, compared to the right upper extremity (Figure 3b). Annual upper extremity electromyogram (EMG) control was done. Earliest signs of nerve functioning appeared after the first year. In the upper extremity, an EMG performed at the end of the fifth year confirmed full innervation in the motor and sensory branches of the radial nerve and the opposite side nerve conduction velocities showed a similar functional range. The patient and relatives were satisfied with the postoperative outcomes and there was no significant difference between the opposite side of the extremity in the daily active use of the elbow and wrist follow ups.

A written informed consent was obtained from the legal guardian of the patient for the publication of this case report.

\section{DISCUSSION}

In our case, radial nerve transection after pediatric open supracondylar humerus fracture can achieve excellent results with a good microsurgery and primary repair after fracture fixation.

About 10 to $20 \%$ of supracondylar humerus fractures result in a nerve injury, most commonly median nerve neurapraxia. Radial nerve injury accounts for 5 to $29 \%$ of these neural injuries. ${ }^{[6]}$ Early complications of supracondylar humerus fractures usually occur, due to tissue injury due to direct blunt trauma, tear injury caused by traction of sensitive tissues, and fractured bone injuries of neurovascular structures. Although all these complications can occur with any type of supracondylar humerus fracture, specific types of injuries tend to damage certain tissues. 
Posterolateral displacement of extension fractures has a tendency to injure the brachial artery and median nerve, whereas posteromedial displacement of extension fractures usually injure the radial nerve and flexion types tend to injure the ulnar nerve ${ }^{[4]}$ Our case was a radial nerve injury occurring after a posteromedial displacement of an extension supracondylar humerus fracture.

Possible indications for nerve exploration in the presence of post-traumatic neurological deficit, inability to achieve acceptable reduction in the fracture with nerve injury after open or closed fixation without direct visualization of the nerve, complete loss of nerve function with sympathetic paralysis, progressive neurological deficit and accompanying ischemia within 8 to $12 \mathrm{~h} \cdot{ }^{[7]}$ Open reduction and nerve exploration are usually not necessary in the presence of early accompanying with neurological damage in a closed fracture, and spontaneous healing is observed in an average of two to six months. Accompanying nerve transection is extremely rare and is mostly seen in the radial nerve. ${ }^{[7]}$

Previous reports of some neurological complications identified during postoperative examination could represent complications caused by the trauma itself that did not occur during the operation. ${ }^{[8]}$ A previous study of 100 cases of supracondylar humerus fracture revealed that $16 \%$ of the patients had nerve injury, and yet only $3 \%$ of those patients had an iatrogenic nerve injury. ${ }^{[9]}$ The aforementioned study also reported that the nerve injury incidence increased with higher Gartland criteria, with type II at $7 \%$, type III at $19 \%$, and type IV at $36 \% .{ }^{[8]}$ These findings and our case reveal the importance of the preoperative physical examination and attentive wound exploration in light of the physical findings, particularly in cases with open fractures and higher Gartland criteria.

A study conducted by Abu-Jayyab et al. ${ }^{[9]}$ showed that functional and cosmetic results were good in both the elbow and wrist in an unstretched primary repair of the radial nerve total injury, which occurred after a humerus lateral condyle and medial epicondyle fracture dislocation. Primary repair should be done without tension; otherwise, a sural nerve graft should be done. ${ }^{[10]}$ In our case, the unstretched primary repair of the radial nerve injury occurred after the humeral supracondylar fracture was performed, and good functional and cosmetic results were obtained. Since our primary repair was not under tension, we did not need a nerve graft.
In conclusion, it should be kept in mind that after a pediatric supracondylar humerus fracture, vascular and nerve injuries may accompany, depending on the displacement direction of the fracture. Therefore, a good surgical planning should be done. To obtain a functional and cosmetic good result with the microsurgical repair of the radial nerve injury, which is rare after humerus supracondylar open fracture, appropriate antibiotic prophylaxis and good washing and debridement of the surgical area and stability of fracture fixation, as well as unstressed microsurgical repair of the radial nerve are essential.

\section{Declaration of conflicting interests}

The authors declared no conflicts of interest with respect to the authorship and/or publication of this article.

\section{Funding}

The authors received no financial support for the research and/or authorship of this article.

\section{REFERENCES}

1. Erhart S, Lutz M, Larcher P, Blauth M, El Attal R. A case of supracondylar humerus fracture with vascular impairment. Open it up? Arch Orthop Trauma Surg 2013;133:835-9.

2. Leitch KK, Kay RM, Femino JD, Tolo VT, Storer SK, Skaggs DL. Treatment of multidirectionally unstable supracondylar humeral fractures in children. A modified Gartland typeIV fracture. J Bone Joint Surg [Am] 2006;88:980-5.

3. Vaquero-Picado A, González-Morán G, Moraleda L. Management of supracondylar fractures of the humerus in children. EFORT Open Rev 2018;3:526-40.

4. Gosens T, Bongers KJ. Neurovascular complications and functional outcome in displaced supracondylar fractures of the humerus in children. Injury 2003;34:267-73.

5. Hoff WS, Bonadies JA, Cachecho R, Dorlac WC. East Practice Management Guidelines Work Group: update to practice management guidelines for prophylactic antibiotic use in open fractures. J Trauma 2011;70:751-4.

6. Kwok IH, Silk ZM, Quick TJ, Sinisi M, MacQuillan A, Fox $\mathrm{M}$. Nerve injuries associated with supracondylar fractures of the humerus in children: Our experience in a specialist peripheral nerve injury unit. Bone Joint J 2016;98-B:851-6.

7. Ramachandran M, Birch R, Eastwood DM. Clinical outcome of nerve injuries associated with supracondylar fractures of the humerus in children: The experience of a specialist referral centre. J Bone Joint Surg [Br] 2006;88:90-4.

8. Joiner ER, Skaggs DL, Arkader A, Andras LM, LightdaleMiric NR, Pace JL, et al. Iatrogenic nerve injuries in the treatment of supracondylar humerus fractures: Are we really just missing nerve injuries on preoperative examination? J Pediatr Orthop 2014;34:388-92.

9. Abu-Jayyab Z, Abu-Zidan F, Marlovits S. Fracture dislocation of the lateral condyle and medial epicondyle of the humerus associated with complete radial nerve transection. J Pak Med Assoc 2011;61:920-1.

10. Ogawa BK, Kay RM, Choi PD, Stevanovic MV. Complete division of the radial nerve associated with a closed fracture of the humeral shaft in a child. J Bone Joint Surg [Br] 2007;89:821-4. 